\title{
Gribiche, ou la leçon de choses Note pour une théorie de l'allégorie au cinéma
}

\author{
Marc-Emmanuel Mélon \\ Centre de recherche sur les Arts du Spectacle, le cinéma et les arts visuels \\ Université de Liège
}

Référence de cet article (mention obligatoire) :

Mélon, Marc-Emmanuel, «Gribiche ou la leçon de choses. Note pour une théorie de l'allégorie au cinéma», in 1895. Revue de l'Association française de recherches sur l'histoire du cinéma, $\mathrm{n}^{\circ}$ hors-série «Jacques Feyder», sous la direction de Jean A. Gili et Michel Marie, Paris, AFRHC, octobre 1998, pp. 73-98.

«Nous manions une machine à digérer l'univers; il nous faut la nourrir de miettes, d'épluchures...»

Jacques Feyder ${ }^{1}$

Sans réduire à un pur schéma la diversité du champ cinématographique français des années 1920, l'histoire classique considère traditionnellement que la production de cette période se partage en deux grandes tendances. La première est majoritaire et industrieuse. Dominée par des impératifs de production, elle réalise des films de facture conventionnelle, le plus souvent à partir d'adaptations d'œuvres littéraires reconnues. Sa légitimation, essentiellement économique, lui est reconnue par le succès populaire des films qu'elle produit. La seconde, dite d'«avant-garde» poursuit, avec des différences importantes, le projet parnassien de l'Art pour l'Art. Elle est le fait d'une minorité d'individus qui se reconnaissent mutuellement, ont des revendications de modernité, d'innovation, de subjectivité et d'étanchéité de l'artiste, coupé des réalités du monde. Sa légitimation est symbolique et lui est donnée par la reconnaissance des pairs et de l'élite intellectuelle. Elle s'identifie aussi, négativement, par le refus de l'uniformisation collective, de l'industrialisation et de l'art comme marchandise. Cette distinction toute théorique, qui rend peu compte des réalités économiques de la production (beaucoup moins tranchées), a pour conséquence de rejeter très souvent dans les poubelles de l'histoire toute une production trop rapidement condamnée pour «noncinématographicité» comme les fameuses (et si décriées) adaptations littéraires (de Jacques de Baroncelli par exemple), ou encore les films réputés mineurs d'un grand cinéaste, comme Le lion des Mogols de Jean Epstein, et qui constituent cependant d'importants jalons de l'histoire culturelle française. Elle a aussi pour effet d'«oublier» les films de Jacques Feyder, qui n'ont plus fait l'objet d'une étude historique approfondie depuis la monographie de Charles Ford, il y a vingt-cinq ans².

Peu disposé au formalisme ou aux effets visuels gratuits, Feyder a subi le contrecoup d'une histoire du cinéma trop préoccupée par l'édification de l'art cinématographique et donc encline à récupérer à son avantage les catégories esthétiques en vigueur dans le système des beaux-arts. A la différence des films de Gance, L'Herbier ou Epstein qui, par le fécond renouvellement des formes 
visuelles qu'ils proposent, pouvaient être estimés à la mesure de ces catégories, qu'en retour ils transformaient, les films de Feyder, jugés à l'aune de ces mêmes valeurs et en recourant aux mêmes concepts esthétiques sans que leur validité soit remise en cause, étaient aussitôt ravalés au second rang. Pierre Billard synthétise parfaitement cette incapacité de l'histoire classique à renouveler ses propres catégories en fonction de la spécificité de ses objets, lorsqu’il écrit : «Feyder n’est sans doute pas un auteur porteur d'un univers» ${ }^{3}$.

Feyder, dans cette perspective, aurait tout du cinéaste «moyen», à mi-chemin entre les avantgardes novatrices qui cherchent à dépasser les limites mêmes du cinéma, et les suppôts de l'industrie, faiseurs de cinéromans et de feuilletons à épisodes ou médiocres adaptateurs des chefs-d'œuvre de la littérature. Il ne trouverait sa place qu'«en marge de l'impressionnisme» (Sadoul), dans un filon «réaliste» qui irait de Feuillade à Carné. Ce malaise n’est pas nouveau. Le cinéma essentiellement narratif d'un réalisateur non théoricien a depuis toujours indisposé les commentateurs qui se perdent dans des circonvolutions de langage ou sombrent dans la condescendance. Henri Fescourt, observateur nuancé du cinéma de son temps, écrit : «Jacques Feyder résiste au plaisir de reproduire un tableau sous des aspects photographiques multiples, d'insister sur le visuel. (...) S'ensuit-il qu'il méprise l'image en soi ? L'image pour l'image, c'est probable. Mais celle qu'il choisit n'est jamais la première venue.» ${ }^{4}$ Dans un hommage à Feyder publié après sa mort en 1949, Nino Frank est plus clair : «Je le tiens pour un artisan et non pour un artiste, un technicien et non un auteur.» ${ }^{5}$ L'artisan, voilà le bon terme, le «juste milieu» qui caractérise si bien le cinéaste que Victor Bachy l'utilisera dans le titre de sa monographie ${ }^{6}$. Du reste, le mot vient de Feyder lui-même qui n'a cessé de se désigner ainsi, avec une humilité qui l'honore : «Je suis un ouvrier, je me suis débrouillé comme j’ai pu, j’ai mis la main à la pâte, je suis un artisan au sens plein du mot, honorable et limité.» ${ }^{7}$ Dans le champ intellectuel bouillonnant des années 1920, son silence et l'absence apparente de préoccupations théoriques laissent Feyder à l'écart du club assez fermé «des amis du septième art». Il se défend, d'ailleurs, d'avoir contribué d'une quelconque façon à une «métaphysique» du cinéma : «L'auteur de ces souvenirs n'est ni un esthéticien, ni un critique, ni un philosophe. Il a, jusqu'à ce jour, travaillé sans arrière-pensée, sans méditation de l'origine, de l'essence et des buts que se propose l'art qu'il exerce. Il a toujours résolu, au fur et à mesure, les problèmes où l'engageait chacune de ses œuvres; il n’a pas cherché les lois générales qui y commandent la création.» ${ }^{8}$ Charles Spaak est encore plus clair : «Jacques Feyder avait horreur des abstractions, des vues obscures, des mots savants, de toutes les sottises que l'on écrit abondamment sur les fins et les moyens intrinsèques du cinéma.»" "Un noble artisan, conclut un peu vite Charles Ford, ne s'embarrasse pas de théories inutiles.» ${ }^{10}$

Un tel débat, qui n'a plus guère de sens aujourd'hui, témoigne cependant de l'inadéquation des classifications traditionnelles fondées sur les notions équivoques d'«art» ou d'«auteur», que ceux qui y ont recours affectionnent justement pour leur ambiguité, et qui conduisent les commentateurs à juger par la négative les films ou les réalisateurs qui n'entrent pas dans ce genre de catégories. Le cas Feyder est donc intéressant à analyser dans l'une ou l'autre perspective qui tendrait à remplacer ces notions impropres par de nouvelles plus adéquates parce que indépendantes du système de valeurs déjà mis en place à l'époque où les films furent réalisés. Aujourd'hui, l'un des principaux enjeux de l'histoire du cinéma est certainement de rendre à certaines productions «oubliées» par l'histoire classique (pour non conformité aux catégories esthétiques qu'elle avait elle-même élaborées) leur place réelle dans l'histoire, et donc de forger les notions théoriques adéquates pour pouvoir en rendre compte. Car c'est aussi le travail de l'histoire de ne pas se résumer à collecter des faits pour en dresser l'inventaire, mais de proposer de nouvelles classifications sur la base de critères inédits, de constituer des séries originales, de découvrir d'autres similarités et d'autres différences. Pour restructurer la nébuleuse du cinéma français des années 20 dans des ensembles qui prennent en compte l'étendue et la diversité de la production, sans exclure ni jeter d'anathème sur des films qui neseraient pas 
conformes à certaines conceptions actuelles du cinéma, le critère que l'on voudrait ici proposer est celui de la production du sens.

Face à la très ancienne question du rapport entre l'image et le sens, le cinéma français des années 20 semble en effet avoir été partagé entre deux attitudes possibles : soit produire un sens nouveau, que seul le cinéma serait en mesure de créer, soit reproduire un sens donné, déjà présent au monde, mais que le cinéma serait en mesure de désigner, peut-être de transformer. Dans le premier cas, le cinéaste exploite l'image comme une matière, une glaise qu'il pétrit, malaxe, sculpte pour en tirer des «figures» dont la nouveauté (grain, «photogénie», mouvement, rythme, vitesse) permettrait de faire surgir un sens supérieur, indéterminé, non traduisible en mots mais assez puissant pour entraîner l'homme au-delà de lui-même, au-delà des contingences de l'Histoire et donc toucher une sorte de transcendance sublime. Par un traitement intense de la plastique de l'image, de sa musicalité (la fameuse «symphonie visuelle» de Gance et Dullac), le cinéaste fait de ce travail même, et des sensations qu'il procure, l'enjeu suprême de la réalisation. Les films qui en résultent, parce qu'ils touchent à l'essence même de l'image et posent des questions fondamentales à la représentation, visent une valeur supérieure et éternelle, que l'histoire du cinéma, pour cette raison même, leur a déjà reconnue. Ce sont ces films qui, aujourd'hui plus que jamais, font l'objet d'une attention assidue de la part de certains esthéticiens et théoriciens du cinéma, pour lesquels le sens est indissociable de l'image qui le produit et qu'elle contient intrinsèquement ${ }^{11}$.

Dans le second cas, l'image est traitée non pas comme une matière mais comme un signe, que le film incorpore dans une nouvelle représentation du monde. L'image-signe, qui se définit ainsi par l'écart qu'elle inscrit avec son objet, travaille les rapports entre les choses, édifie éventuellement de nouvelles structures. Elle entre dans une série signifiante et constitue un discours que le film a charge de communiquer. Par ce travail, par cet imaginaire, par la façon dont il reconstitue et donc transforme l'ordre des choses, mais aussi par la conscience qu'il a du discours qu'il énonce, le film donne un sens au monde. Mais l'idée qu'il exprime reste ancrée dans l'histoire des hommes dont elle est tributaire, n'est pas éternelle. Le film est dès lors soumis à son historicité fondamentale, à sa réalité de fait, inscrite dans l'Histoire où le sens qu'il désigne est condamné à l'oubli, sauf à l'en faire resurgir par le travail historique. On l'a compris, la distinction qui est ici proposée est aussi essentielle que celle qui oppose, dans l'histoire de la pensée, l'idéalisme et l'historicisme. C'est la différence entre une conception verticale du rapport entre l'image et le sens, où celui-ci, comme les Idées platoniciennes, est insaisissable, sauf à s'incarner dans l'image, laquelle n'a de sens qu'en elle-même et pour elle-même, un sens transcendant et éternel; et une conception borizontale où le sens, historique et éphémère, ne se constitue que dans un rapport empirique entre l'image et ce qu'elle représente, en vertu du principe phénoménologique selon lequel «l'image est toujours image de quelque chose» (Jean-Louis Baudry ${ }^{12}$ ).

Pour abstraite et conceptuelle qu'elle puisse paraitre, la distinction ici proposée s'inscrit assez nettement dans l'histoire des images où elle correspond à deux très anciennes catégories esthétiques, depuis toujours opposées l'une à l'autre, et qu'il faut à présent désigner : le symbole et l'allégorie. La première attitude décrite, qui correspond assez clairement à certains films d'avant-garde (L'Inbumaine, La chute de la maison Usher, La Coquille et le Clergyman, par exemple), peut être dite «symboliste»; la seconde, qui ne s'identifie pas aussi simplement à la grande production industrielle (même si les films qui entrent dans cette catégorie en proviennent pour la plupart) sera dite, par parenté conceptuelle, «allégoriste» - terme qu'il faudra définir. C’est de cette seconde tendance que relèvent aussi bien J'accuse et Napoléon d'Abel Gance, que Les Misérables d'Henri Fescourt et la plupart des films de Jacques Feyder, en particulier Gribiche qui sera analysé plus loin, mais aussi Crainquebille, Visages d'enfants, L'Image et Les Nouveaux Messieurs, y compris certains films réalisés dans les années 30 (Le Grand Jeu, La Kermesse béroïque ou La loi du Nord). 
Sans du tout oublier l'importance des facteurs de production et des contraintes techniques, idéologiques, sociales et économiques qui restent déterminants, la distinction théorique ici proposée ne permet pas seulement de créer de nouveaux ensembles de films, qui ne doivent plus rien à la notion d'avant-garde, ni aux anciennes classifications, devenues obsolètes, telles que «art vs industrie» ou «auteur vs artisan». Elle permet surtout, par sa souplesse, de ne pas constituer des ensembles compacts et rigides. Tout film, à la limite, selon le point de vue d'où on l'observe, pourrait être introduit dans chacune des deux catégories. La Roue, par exemple, selon qu'on le considère sur le plan de ses recherches formelles, de son appartenance au genre mélodramatique, ou des représentations historiques de sexe, relève de l'une et de l'autre. Il ne s'agit plus, aujourd'hui, comme Emile Vuillermoz l'avait écrit à l'époque, de demander à Abel Gance de choisir entre deux voies, celle du «succès populaire» ou celle de la «conquête de l'élite», et de couper dans son film...

Gribiche semble particulièrement représentatif de cette tendance «allégoriste» du cinéma français des années 20. Relativement peu connu et commenté, il fait partie de ces films considérés comme mineurs par l'histoire de l'art cinématographique. Adaptation d'un «cinéroman» de Frédéric Boutet ${ }^{13}$, il porte sans doute la marque d'une sentimentalité bonne enfant et un peu mièvre, propre au mélodrame. En même temps, c'est un film qui témoigne d'un sens de l'observation très aigu et qui dresse des classes sociales un tableau d'une grande finesse, bien loin des stéréotypes habituels. Il a, de ce point de vue, une dimension pédagogique certaine, qui passe par une utilisation judicieuse et une articulation très élaborée des signes visuels, invitant le spectateur à regarder autrement la culture de classe, et donc à s'y retrouver.

En mai 1925, Jacques Feyder, qui vient de terminer L'Image, rencontre Alexandre Kamenka, de la compagnie Albatros, avec lequel il signe un contrat pour la réalisation d'un long métrage. C'est le cinquième film du réalisateur et le premier qu'il réalise pour la compagnie de la «colonie russe» à Paris qui regroupe, autour de Kamenka, des producteurs, réalisateurs, acteurs et techniciens émigrés en France après la Révolution d'Octobre, parmi lesquels Ermolieff, Mosjoukine, Protazanoff, Tourjansky, Volkoff, Lochakoff, Bilinsky et un certain Lazare Meerson ${ }^{14}$. Dès le début, Kamenka a compris qu'il devait ouvrir les studios de Montreuil à des réalisateurs français et étrangers : Henri Etiévant, Robert Boudrioz, Jean Epstein, Marcel L'Herbier, René Clair, Gustav Molander et enfin Jacques Feyder qui, après Gribiche, réalisera encore deux autres longs métrages pour la compagnie, Carmen en 1926 et Les Nouveaux Messieurs en 1928. Pour les trois films, Feyder tournera avec deux excellents techniciens de la maison, Maurice Desfassiaux comme opérateur (qui, avec Maurice Forster, signe notamment de remarquables vues nocturnes, à la lumière des réverbères) et, pour la première fois, Lazare Meerson comme décorateur.

A première vue, Gribiche semble relever du mélodrame dont il hérite des données génériques et d'un argument narratif à la fois très conventionnel et invraisemblable. Dans un grand magasin chic où il se rend pour acheter un cadeau à sa mère, Gribiche (Jean Forest), un gamin de Paris jovial, débrouillard et généreux, découvre un sac à main qu'une dame (Françoise Rosay) vient de laisser tomber. Il la rattrape, lui rend son bien, refuse le billet qu'elle lui tend. Gribiche vit avec sa mère (Cécile Guyon), une jeune veuve de guerre, ouvrière en usine, dans un petit appartement du quartier populaire de Grenelle. La dame, Edith Maranet, une Américaine veuve d'un diplomate français, appartient à la haute société. D'allure hautaine et snob, elle ne cache pas le luxe dans lequel elle vit. Elle se consacre à des cuvres d'hygiène sociale et dirige une pouponnière où elle donne des conférences. Un samedi soir, de sortie en ville avec sa mère et le contremaître de l'usine, Gribiche comprend qu'il est devenu un obstacle à leur mariage. Le lendemain matin, Edith Maranet propose à la mère de Gribiche de prendre le garçon chez elle afin de lui donner l'éducation qu'il mérite : «Je 
pense que votre fils a une nature d'élite digne d'intérêt». La mère refuse mais Gribiche accepte. Il découvre alors un autre univers : une grande maison, dans le quartier de l'Etoile, très spacieuse et meublée dans le style «Art Déco» à la mode, une femme d'affaires autoritaire, une société de femmes oisives qui vient prendre le café l'après-midi, et le golf comme seul divertissement. Il découvre surtout un autre mode de vie, protocolaire et sans âme, un horaire journalier strict, des domestiques jaloux et une éducation à la spartiate réglée comme du papier à musique : lever à 6h30, bain, boxe, douche, déjeuner, manucure, leçons de mathématiques, de français et d'anglais données par des professeurs particuliers, promenade au parc. Edith Maranet, persuadée qu'elle fait œuvre de charité, se vante partout qu'elle a sauvé l'enfant de la misère la plus noire. Coupé de sa famille, de ses copains et de la vie de la rue, Gribiche s'ennuie et se réfugie dans le garage où il se passionne pour la mécanique et sympathise avec le chauffeur. Le 14 juillet, frustré de ne pouvoir participer à la fête «parce que ce sont des activités anti-hygiéniques», Gribiche s'éclipse discrètement, va au bal des barrières où il est reconnu par les domestiques qui le poursuivent. Il leur échappe et retourne à Grenelle où il retrouve sa mère, remariée avec le contremaître, qui l'accueille chaleureusement. Edith Maranet, déçue par ce qu'elle croit être de l'ingratitude, déclare à son frère que «seule la charité collective est bonne, la charité individuelle est décourageante, fausse, injuste». Mais à Gribiche venu la remercier avec sa mère, elle offre encore de l'argent «pour son éducation».

Les données narratives de base sont conformes aux lois du genre : deux veuves (une ouvrière et une bourgeoise), deux univers sociaux opposés et un personnage d'orphelin qui va de l'un à l'autre. Cependant, Gribiche échappe aux normes du mélodrame d'une double façon : d'une part, le film est une satire mordante de la société bourgeoise, dont il dénonce la vanité et l'hypocrisie; d'autre part, il brosse un tableau juste et subtil de la culture populaire et de son cadre de vie. Feyder se montre en cela un très fin observateur de la vie sociale, de ses clivages apparents comme de ses ressorts cachés. Si le portrait qu'il croque de la société bourgeoise est, à certains égards, exagéré, c'est pour mieux faire ressortir, par contraste, le réalisme des scènes de la vie populaire, comme Feyder l'a précisé luimême au moment du tournage :

Le sujet, je crois, sera très "public», car il me permet de montrer en même temps les milieux populaires de Paris pendant les fêtes du 14 juillet, les bouillons, les bistrots, les bals de barrière, et aussi le milieu élégant dans lequel évolue une Américaine milliardaire.. ${ }^{15}$

Les scènes ici évoquées sont à la fois des scènes nocturnes et des scènes de foule, comme Feyder les affectionne et dont il maitrise bien la mise en scène. Leur réalisme est dû en grande partie au tournage de nuit. L'entrée de la brasserie, avec son auvent éclairé par les lampes intérieures, la fête foraine filmée du manège, les rues désertes dans le quartier de Grenelle, sous les réverbères, le bal final sous les lampions sont d'incontestables réussites, surtout à une époque où les scènes nocturnes sont généralement filmées de jour et teintées en $\mathrm{bleu}^{16}$. Ces performances techniques, et les moyens importants qu'elles ont réclamés, confirment le souci de Feyder d'exploiter les atouts du réalisme pour toucher le public le plus large possible et obtenir ainsi, tout simplement, qu'il s'y reconnaisse. S'il y a sans aucun doute derrière cette intention une idée de marketing, il y a aussi le souci d'adopter, pour son film, le point de vue du public, qui se confond ici avec celui de Gribiche.

L'un des moyens que Feyder utilise pour construire sa satire consiste à créer un écart très perceptible entre l'image et le discours (y compris dans sa dimension narrative). Le discours, dans Gribiche, c'est l'expression de la bonne conscience bourgeoise qui s'incarne explicitement dans le personnage d'Edith Maranet dont la parole, d'un bout à l'autre du film, est la principale activité. Elle cause, converse, pérore et écrit des lettres à son frère dans lesquelles elle s'épanche sur la charité. 
Cette assimilation du personnage au discours — et notamment au discours édificateur — est établie dès le départ, lorsqu'on voit Mme Maranet donner une conférence sur les bienfaits de la stérilisation des biberons. Assise devant des affiches sur lesquelles on peut lire «Suivez ces conseils, vous vivrez longtemps», l'air doctoral, les lunettes sur le nez, le doigt levé, elle parle en ar-ti-cu-lant et en soulignant chaque mot d'un petit geste de la tête. L'ironie de Feyder apparaît quand il montre le public qui écoute l'oratrice, composé de bourgeoises âgées, portant lorgnons et appareils acoustiques. Cette inadéquation comique entre le public et l'objet de la conférence souligne l'inanité du discours tenu par la conférencière qui, simple moulin à paroles, fait tourner la machine de l'hygiène «morale».

L'intention satirique du film est plus explicite dans les séquences bien connues au cours desquelles, à deux reprises, Mme Maranet raconte comment elle a rencontré Gribiche et l'a sauvé de la faim et de la misère la plus noire. Chacun de ces récits rapportés, traité comme une parodie de mauvais mélo, déforme la réalité en noircissant gaiement la situation. Un seul intertitre préalable annonce que le récit sera transformé par la vision subjective : «Dans son souvenir, les événements se modifiaient à son insu». On voit ensuite Gribiche en guenilles, mourant de faim devant la vitrine bien garnie d'une boulangerie. Il ramasse le sac à main qu'Edith Maranet vient de laisser tomber, découvre les nombreux billets qui s'y trouvent, regarde les pains et les croissants dans la vitrine et, d'un geste grandiloquent feignant le désintéressement, rapporte le sac à sa propriétaire. Le second récit montre la mansarde sordide où la mère de Gribiche, atteinte de tuberculose, crache ses poumons. Mme Maranet entre, prend pitié, offre de l'argent et s'en va avec l'enfant ${ }^{17}$. Le spectateur, qui n'ignore pas la «réalité» des faits qui lui a été racontée au début du film, se plait à mesurer l'écart entre le récit premier et le récit second. Le décor de carton et de toiles peintes, le maquillage, le jeu déclamatoire et emphatique soulignent l'affabulation. Cette mise en cause radicale du récit de la narratrice inscrit un écart entre le visuel et le narratif, le premier exprimant le second tout en en dénonçant la subjectivité et la facticité. Par cet écart, par la théâtralité excessive et baroque du jeu d'acteurs, par sa spectacularité et son artifice, par la défiguration des choses, ici explicite, toute la scène dissipe l'illusion, brise le rêve naïf et dérisoire d'un monde harmonieux où régnerait la vertu et la bonne conscience.

Le film acquiert ici, de manière très convenue, une allure pédagogique. Il s'agit de former le regard du spectateur et de l'orienter vers ce que le film veut signifier. La mise en cause du récit par l'image invite le spectateur à séparer ce qui s'énonce de ce qui se voit et à décoder les signes qui lui sont transmis. Comme le film dépeint deux univers que tout oppose (par le recours presque constant au montage alterné), la comparaison constitue l'essentiel de l'activité spectatorielle. Mais ce processus est ici dirigé de manière à faire voir ce que, sans cela, le spectateur ne verrait pas : l'univers «réel» dans lequel vit Gribiche, qui n’est pas extraordinaire, mais n’est pas banal non plus.

Cette opération de désignation qui pointe son objet, l'encadre et attire sur lui l'attention du spectateur, est relayée explicitement dans le film par le jeu des regards des deux protagonistes principaux, Gribiche et Edith Maranet, observateurs attentifs de l'univers de l'un et de l'autre. La première scène du film se déroule aux Magasins des Trois Quartiers, près de la Madeleine, un des célèbres grands magasins parisiens qui constituaient, depuis le XIXe siècle, la façade la plus visible du capitalisme bourgeois ${ }^{18}$. Dès le second plan, la caméra accompagne la foule, de dos, qui se précipite sur les soldes, en un mouvement de forte pénétration vers l'avant entraînant dans son sillage le regard du spectateur. Ensuite, c'est à travers les yeux amusés de Gribiche que la caméra circule dans le magasin. Appuyé contre un comptoir, le gamin observe les mannequins curieusement accoutrés ou encore le manège d'une femme qui fouille un étalage de chapeaux. Gribiche devient ici l'observateur attentif du commerce bourgeois. En marge d'un monde auquel il n'appartient pas (il ne se trouve dans ce magasin qu'en raison des soldes), il occupe une position comparable à celle de Charlot (le misérabilisme en moins), notamment dans The Floor-Walker (Charlot chef de rayon, 1916). C'est aussi la 
position baudelairienne du flâneur, dont la passion est «d'épouser la foule. (...) Être hors de chez soi, et pourtant se sentir partout chez soi; voir le monde, être au centre du monde et resté caché au monde (...) L'observateur est un prince qui jouit partout de son incognito.» ${ }^{19}$

À l'inverse, lorsque Mme Maranet se rend chez Gribiche, dans le quartier de Grenelle, elle commence par jeter un coup d'œil aux alentours : l'étal du marchand de légumes d'un côté, la teinturerie de l'autre. Ainsi le film associe-t-il le monde de Gribiche au petit commerce, et celui d'Edith Maranet aux grands magasins. Lorsqu'elle pénètre dans l'appartement, accueillie par la mère en peignoir, elle balaie du regard le décor de la pièce et les objets posés sur la cheminée qui acquièrent ainsi, sous son regard, une valeur de signe, ou d'indice. Pendant qu'elle discute avec Gribiche et sa mère, des gamins de rue s'approche de la Rolls Royce, narguent le chauffeur et se moquent du petit chien à col de fourrure. A leurs yeux, ces marques visibles de la classe possédante sont des objets de curiosité aussi exotiques que les animaux d'un zoo. De même, lorsque Gribiche pénètre dans la maison luxueuse du quartier de l'Etoile, un regard furtif isole une statuette, un autre l'immense suspension en forme de fontaine. Ces jeux de regard, qui introduisent les plans subjectifs, fonctionnent comme des interpellations du regard spectatoriel, des invitations à observer les objets, qu'ils soient d'apparence insignifiante ou insolites, et à les décoder comme autant de signes culturels visibles. Le décor et les accessoires qui le constituent deviennent ainsi des outils de désignation, des emblèmes en quelque sorte de la culture qu'ils représentent (et donc, comme on le verra plus loin, les instruments essentiels du discours allégorique).

Dans son ouvrage consacré à la production Albatros, François Albera analyse le film de Feyder en insistant sur le rôle essentiel du décor dessiné par Lazare Meerson, en particulier pour la maison luxueuse et moderne de Mme Maranet ${ }^{20}$. Meerson meuble l'espace aux formes pures et spacieuses avec des productions des décorateurs Louis Süe et André Mare, les deux maîtres d'œuvre de la grande Exposition des Arts Décoratifs qui se tenait à Paris au moment même où Feyder tournait son film. Albera note très justement que, dans la mouvance hétérogène des Arts Décos, Süe et Mare représentaient une tendance élitiste produisant de façon artisanale des produits de luxe destinés aux couches fortunées de la société. Pour lui, l’opposition qui structure le film, marquée plastiquement par la différence entre l'intérieur «Art Déco» de l'Américaine et l'appartement «banal» de la mère de Gribiche est moins sociale (bourgeoisie vs classe populaire) que culturelle (américanisme vs culture populaire). Si Albera a raison de dire que l'opposition qui fonde le film est culturelle (on va y revenir dans un instant), elle n'en est pas moins sociale et l'américanisme n'a rien à voir dans cette affaire. Une brève digression sur les origines historiques et idéologiques des Arts Décoratifs est ici nécessaire.

L'Art Déco, en effet, ne naît pas avec l'Exposition de 1925, qui en sonne plutôt le glas, et ne relève en rien de la culture américaine, mais bien de la vogue bourgeoise et européenne de la décoration qui remonte au milieu du XIXe siècle, avec les écrits de William Morris et de John Ruskin, et plus tard d'Emile Van de Velde. Ces représentants d'une bourgeoisie «progressiste» avaient élaboré les premières bases d'une conception sociale de l'art, projetant de créer industriellement mais aussi artistiquement des meubles et des objets usuels pouvant être acquis par le peuple (on reconnaît dans ce projet très paternaliste une certaine proximité avec les nobles intentions hygiénistes et charitables de Mme Maranet). Ce qu'on appelait alors les «arts décoratifs et industriels» se sont partagés en deux grandes chaînes de production, l'une plus artisanale fabriquant des objets de luxe, de style moderne, destinés à la bourgeoisie fortunée, l'autre, industrielle, fabriquant en série des meubles dits «de style» — du néo-renaissance au néo-Louis XVI, encore très à la mode dans les années 1920 — et destinés aux couches populaires. Les deux chaînes partageaient donc le même credo «moderniste» : fusion des beaux-arts et des arts appliqués, fabrication en série, transformation de l'objet quotidien en objet d'art, création d'ensembles décoratifs cohérents englobant tous les métiers, 
de l'architecture de la maison au flacon de parfum. Entre le palais couvert de marbres et de mosaïques et la cité ouvrière sobre et aérée, une même idéologie circule : celle d'une vision globalisante de l'environnement intérieur, tout entier unifié sous un même style. Cette conception totalisante de l'art décoratif, née au XIXe siècle, atteint son apogée avec l'Art Nouveau, dont les grands maîtres - Horta le premier — traduiront plastiquement, par la fusion complète des formes et des matières, cette vision unifiée de la décoration et de l'architecture. L'abandon, ensuite, de la ligne ondoyante et végétale au profit de la droite et d'une structure géométrique apparente ne constitue en rien un changement de concept mais seulement un renouvellement du style qui commence dès les années 1905-1906. Cette évolution est d'abord le fait de la Sécession de Vienne et de l'architecte Josef Hoffmann qui, en 1905, construit à Bruxelles le Palais Stoclet dont le décor, à la fois riche et sobre, est très proche de celui que Meerson construit pour le film de Feyder. Le style se répand ensuite dans l'Europe de l'Est, jusqu'à Saint-Petersbourg (où peut-être Meerson l'avait rencontré avant d'émigrer), puis en France où il subit l'influence de l'Art Nègre et du Cubisme. Ce qu'on appelle aujourd'hui le «style Art Déco» est donc le fruit d'une longue évolution de plus de vingt ans qui s'achève avec l'exposition de Paris, sans que jamais ait été remise en cause la notion même d'«arts décoratifs» (ce que feront par contre Le Corbusier en France, le Bauhaus en Allemagne et les Constructivistes russes dès le milieu des années 20) ${ }^{21}$. En d'autres termes, le style Art Déco du décor de Meerson est l'expression, remise au goût du jour, d'une conception ancienne et bourgeoise de la décoration. Il est beaucoup moins «moderniste» qu'il en a l'air, il est seulement «moderne»au sens premier du terme : à la mode.

L'intérieur Art Déco d'Edith Maranet d'un côté et la mansarde misérabiliste décrite par ses «souvenirs» déformés de l'autre constituent les deux volets symétriques et radicalement opposés qui, comme le Paradis et l'Enfer dans les triptyques du XVe siècle, encadrent le panneau central, celui de la vie «terrestre» et du destin historique des individus. Ce panneau central a lui aussi son décor, ses acteurs et ses gestes que Feyder expose avec finesse et un sens aigu de l'observation. A plusieurs reprises, le film nous montre en détail l'appartement de Grenelle, dans des plans qui n'ont guère de justification narrative. Le premier montre Gribiche d'abord, sa mère ensuite, se recoiffer et ajuster leur tenue en se regardant dans le miroir ovale de la lingère. Ils sortent de la pièce et la caméra demeure un instant sur le miroir qui fait voir un angle de la chambre. Ce surcadrage interpelle le regard du spectateur et la disposition très apprêtée des meubles dans le champ ouvert par le miroir l'invite à observer avec attention l'aménagement des lieux. Le plan suivant montre la salle de séjour, que Gribiche et sa mère traversent pour sortir. Ces plans ne se justifient qu'à exposer le décor de cet intérieur modeste, avec son buffet néo-renaissance, son lit Louis-Philippe, son guéridon 1900, sa pendule rococo et son lustre en laiton doré néo-Louis XVI, ses gravures et ses photos sur les murs aux papiers peints à rayures ou à gros motifs floraux. Ce décor, qui ne doit rien au goût du jour, n'est cependant pas moins «moderniste» que celui de la maison de Mme Maranet, puisqu'il est un pur produit de l'industrie «décorative», version populaire. Mais il est aussi, par son caractère hétéroclite, à l’opposé de l'idéologie des Arts Décoratifs unifiés. La culture dont il procède est celle de la diversité et de la liberté, restreinte mais effective, de pouvoir choisir, dans la panoplie des objets manufacturés que le commerce propose, ceux qui plaisent et qui conviennent. Certes, ces objets de facture industrielle sont stéréotypés mais c'est leur assemblage dans cet intérieur modeste qui devient l'expression d'un choix, d'une différence, d'une singularité. Ce choix s'exprime notamment dans certains petits détails qui, malgré leur conformité aux usages, apparaissent comme autant de marques de distinction : la broderie posée sur le manteau de la cheminée, le coffret sur le guéridon près du réveil, les éventails au-dessus du lit de la mère ou encore, dans la chambre de Gribiche, les photos de stars de cinéma et une couverture de Ciné-Miroir montrant Charlot dans The Kid. Cette diversité élective et ce mélange des styles sont le produit de toute une histoire familiale qui, de génération en 
génération, à travers divers mariages et grâce à de petits héritages, a accumulé les meubles et les objets comme autant de traces laissées par la vie. Le travail du temps dans la décoration de l'appartement est encore plus perceptible lorsqu'un court panoramique balaie le miroir de la cheminée entouré de photographies : un jeune homme, une gamine, une jeune femme, un vieillard barbu, toute la généalogie familiale s'affiche sur ce mur et y expose, de façon «décorative», l'histoire de ceux qui vivent là, même si beaucoup sont morts ou absents. Ce qui s'énonce dans cette accumulation de signes, ce n'est pas le temps présent, neuf, unifié et à la mode de la culture bourgeoise trop encline à confondre modernité et nouveauté, c'est l'histoire individuelle, c'est le temps historique de la culture populaire indissociable du vécu et du destin, heureux ou malheureux, de ceux qui la font.

Comme on le voit, de part et d'autre, chaque élément du décor, chaque petit objet se constitue comme un attribut emblématique du personnage. Aux broderie, pendule, chromos, réveil et photos de cinéma de l'appartement de Gribiche correspondent la douche futuriste, le chien à col de fourrure, les tableaux de maitres, les couverts multiples et l'orphèvrerie Puiforçat de la maison d'Edith Maranet. Celle-ci porte des lunettes, Gribiche devra en porter aussi dès son arrivée, comme un signe de distinction. Ce souci du détail signifiant apparaît également dans les diverses scènes de repas, où le motif récurrent de la serviette fonctionne explicitement comme signe d'appartenance sociale. Quand Gribiche, sa mère et le contremaître dînent au restaurant, plusieurs plans d'affilée montrent qu'ils attachent leur serviette à leur col, sous le menton. A l'inverse, chez les bourgeois, on place sa serviette sur les genoux. A la fin du film, Gribiche et sa mère déjeunent «sans cérémonie» chez Mme Maranet. L'enfant fait comprendre à sa mère que, là où ils sont, la serviette ne se porte pas autour du cou. Enfin, lors de la dernière scène où la famille est à nouveau réunie au restaurant, chacun noue sa serviette à son col, sauf Gribiche. «Mets ta serviette, lui dit sa mère, tu vas te tacher». Et Gribiche, en souriant, reprend les bonnes habitudes de son milieu.

Il y a certes un peu de paternalisme dans le regard de Feyder lorsqu'il brosse ce tableau de la culture populaire. Mais il y a aussi l'acuité visuelle, le choix rigoureux des objets, la construction méthodique du sens. On pourrait parler de réalisme, si le mot ne recouvrait pas tant de démarches différentes. Ce serait plutôt, de façon très précoce dans l'histoire du cinéma français, une attention extrême au signe et une conception de l'image comme un lieu d'articulation des objets-signes qui, soigneusement répertoriés et agencés, construisent le discours. Non seulement chaque élément, aussi menu soit-il, contribue au sens général, mais ces détails, finalement en nombre assez réduit, sont choisis, collationnés et épinglés là où ils doivent être. Cette observation méticuleuse, ce soin de collectionneur pourraient être rapprochés du regard d'un entomologiste (bien que l'expression ait déjà été utilisée à propos de Buñuel), ou encore de cet autre grand observateur de la vie moderne, et habile praticien de l'art des signes, le photographe Eugène Atget.

Bien qu'aucun lien historique éventuel ne puisse être démontré (Atget meurt à Paris en 1927, quasiment inconnu, excepté de Man Ray et des surréalistes), il y a néanmoins plus qu'une parenté entre le film de Feyder et l'album de photographies, intitulé Intérieurs parisiens qu'Eugène Atget dépose en 1910 au Musée Carnavalet d'abord, à la Bibliothèque Nationale ensuite. Depuis 1890, Atget a photographié systématiquement les rues de Paris, les vieilles enseignes, les étalages des boutiques et surtout les ornements des anciens hôtels parisiens (portes, balcons en fer forgé, buttoirs, etc.). Il fournissait ses clichés aux illustrateurs, qui s'en inspiraient pour l'arrière-plan de leurs dessins d'humour, aux décorateurs de théâtre et aux éditeurs de livres d'art, en particulier ceux consacrés à l'histoire des arts décoratifs, dans lesquels étaient juxtaposées, sur des planches illustrées, des reproductions d'ornements de différents styles, invitant le lecteur à les comparer. Quoique l'album Intérieurs parisiens n'ait pas été publié mais confectionné par Atget lui-même, son montage s'inscrit dans la même logique. Les photographies qui le composent montrent divers appartements, sans leurs 
occupants, offrant au regard une grande diversité d'objets et de meubles de styles. Mais le montage des planches n'est pas seulement stylistique. Atget compose son album en mêlant astucieusement les catégories sociales, de manière à rapprocher des intérieurs populaires et bourgeois. Il place côte à côte une vue du «Cabinet de travail d'un financier, avenue Elysée Reclus, Champ de Mars», avec sa table Louis XVI et sa bibliothèque surchargée de livres, et une autre d'un «Intérieur d'un ouvrier, rue de Romainville», avec son lavabo en faience, son papier peint à fleurs et ses daguerréotypes au mur. Puis, dans le même appartement, sur la cheminée, la pendule rococo, le miroir, les photos et un énorme réveil accroché en hauteur. Se succèdent la «Petite chambre d'une ouvrière, Rue de Belleville»(collection d'éventails, photos, diplômes de secouriste, calendrier et gros réveil), l'«Intérieur de M. F. agent de change Rue Montaigne», l'«Intérieur de M. A., industriel, rue Lepic», celui de «Melle Sorel, de la Comédie française, avenue des Champs Elysées». Ainsi, la comparaison ne joue plus seulement sur les styles, elle opère sur les couches sociales elles-mêmes, dont elle dévoile les inégalités, ou plutôt les signes de l'inégalité. Vides de toute présence humaine, les photos d'Atget invitent le spectateur à observer chaque image, à relever certains motifs, à les introduire dans des séries paradigmatiques (celle du réveil, par exemple, qui rappelle que, dans les milieux ouvriers, on se lève tôt), à déceler les indices visibles des différences sociales. C'est l'inventaire ainsi dressé qui, par accumulation d'indices soumis à l'observation aiguisée du spectateur, fait sens. Par sa force presque statistique, l'album se transforme en une «leçon de choses» sur la culture populaire, qu'Atget, en le déposant dans les hauts lieux de la culture bourgeoise, offre fièrement à l'élite de la sociétée ${ }^{22}$, donnant de la sorte à son œuvre une vraie dimension politique ${ }^{23}$.

Dans Gribiche, le thème de l'enseignement domine tout le film, où il est assimilé à la culture bourgeoise. Cela commence dès les premiers plans, à la pouponnière, où Edith Maranet donne des leçons d'hygiène. C'est pour lui donner l'éducation qui convient à sa «nature d'élite» qu'elle va chercher l'enfant chez sa mère. La nouvelle vie de Gribiche est totalement organisée en fonction de son instruction, qui comprend aussi bien des activités physiques qu'intellectuelles. Les cours se donnent par plusieurs enseignants, dont le film se moque parfois ouvertement. Lorsque des photos coquines tombent de la poche du professeur de français, celui-ci se justifie en disant : «ce sont les photos de mes nièces qui se destinent à la danse», nouvelle et cinglante mise en cause de la parole professorale. Enfin, à la fin du film, Edith Maranet donne de l'argent à la mère de Gribiche «pour son éducation». A cet enseignement bourgeois, Gribiche résiste comme il résiste à la vie protocolaire qu'on lui impose. Mais le film donne aussi, de manière plus discrète, une version positive de l'instruction, celle que Gribiche acquiert par lui-même : c'est tout ce que Gribiche apprend à force de regarder cet univers qui n'est pas le sien. C'est aussi l'apprentissage de la mécanique auquel il trouve bien plus d'intérêt qu'à ses cours de mathématique. Dans cette opposition, c'est tout un débat sur la pédagogie qui transparaît.

Une telle insistance sur l'éducation n'est pas due au hasard : le film lui-même se veut pédagogique. Mais il opte pour une pédagogie pratique, loin des idées abstraites et plus proche de la vie réelle. Il se donne comme un apprentissage ou plutôt, comme chez Atget, une leçon de choses. Il invite son spectateur à se défier des discours (très "parlants», même dans ce film muet) et, au contraire, à observer le monde, à décoder les signes visuels, à regarder les images pour en tirer, par lui-même, la leçon.

Au moment du tournage, Feyder disait de son film qu'il serait «tout public», indiquant par là qu'il toucherait toutes les catégories sociales. En effet, la leçon du film peut se donner dans les deux sens, selon l'origine sociale des spectateurs. Elle invite le public populaire à se défier des discours et à voir, sur l'écran, une image positive de sa propre culture. Au public bourgeois, elle tente d'ouvrir les yeux sur les réalités sociales. Cet unanimisme, commercialement justifié, conduit le film loin de l'engagement politique d'Atget. Il n'empêche, le fait est là : en collectant, avec rigueur et précision, les 
signes et les traces de la vie quotidienne de la classe populaire comme ceux de la classe bourgeoise, et en les instituant comme autant d'emblèmes, il désigne et expose sur le théâtre cinématographique, ostensiblement, une certaine idée de la culture de classe. Voilà pourquoi Gribiche est une allégorie.

$*$

Pour comprendre ce que le concept d'allégorie peut apporter à l'histoire du cinéma, et notamment à une nouvelle approche du cinéma français des années 20, il faut commencer par distinguer entre l'image allégorique classique, qui a toujours été méprisée par les artistes en raison de son caractère assez frustre et de la fonction édificatrice que l'usage lui assignait, et le concept élaboré par Walter Benjamin dans le cadre de sa théorie moderne de l'allégorie. L'allégorie classique est une représentation schématique qui, à partir de conventions de représentation souvent obscures et figées, associe mécaniquement une image à une idée dite supérieure et universelle, avec laquelle elle ne se confond pas, mais qu'elle illustre terme à terme. C'est sans doute dans les anciens traités d'allégories, tous plus ou moins inspirés par le célèbre Iconologia de Cesare Ripa (1593), que l'on peut trouver la définition la plus explicite :

«Les Images que l'Esprit invente sont les symboles de nos pensées. Elles appartiennent proprement aux peintres et autres semblables ouvriers, qui par les couleurs et les ombrages ont trouvé l'admirable secret de donner un corps à nos pensées et de les rendre visibles.» ${ }^{24}$

L'allégorie (que l'on ne confondra pas avec le symbole - cf. infra) est donc d'abord une idée, conçue a priori, à laquelle il faut donner un «corps», c'est-à-dire une image dans laquelle elle s'incarne. Cette image se compose de plusieurs éléments, dits «emblèmes», qui peuvent entrer dans des constellations diverses, selon les allégories. Pour prendre un exemple connu, la Justice est représentée par une femme qui porte une balance, une épée et un bandeau sur les yeux. La balance est l'emblème le plus ancien de la Justice mais l'épée est aussi l'attribut de la Vaillance et de la Vérité, le bandeau celui de la Mort, l'Ambition, la Cupidité, l'Ignorance, etc. ${ }^{25}$ Tous ces éléments visuels entrent ainsi dans des séries dont les articulations relèvent clairement de conventions fonctionnant sur un mode langagier.

L'espace manque ici pour retracer les grandes étapes de la longue histoire de l'allégorie. Disons seulement qu'elle remonte aux toutes premières formes de représentation (les idéogrammes), se répand dans tout le monde antique où elle représente les dieux et les héros, acquiert au Moyen-Age une fonction didactique et édificatrice, et se développe surtout à l'époque baroque où elle devient l'instrument privilégié de la Contre-Réforme. C'est à ce moment qu'est publié le célèbre Iconologia de Cesare Ripa ( première édition à Rome en $1593^{26}$ ). Il prend la forme d'un lexique des figures (l’Abondance, la Chasteté, la Colère, la Vanité, etc.), classées par ordre alphabétique.

Dès son origine, l'allégorie circule grâce à de vastes réseaux de communication. Dans l'Antiquité, les premières images allégoriques étaient imprimées sur des pièces de monnaie. Utilisant ensuite les moyens de l'imprimerie et de la gravure sur bois, elle connaît une large diffusion favorisée par la Contre-Réforme. Le recueil de Ripa circule à travers toute l'Europe des XVIIe et XVIIIe siècles. Il est non seulement l'objet de nombreuses rééditions et traductions, mais aussi le modèle de plusieurs ouvrages qui s'en inspirent explicitement ${ }^{27}$. A l'opposé de la peinture dont les tableaux uniques sont, à l'époque baroque, essentiellement destinés aux lieux de culte ou à l'habitation privée, l'allégorie se définit comme une image «véhiculaire», pourrait-on dire, par analogie au langage. Cet 
usage «médiatique» de l'allégorie l'inscrit plutôt dans l'histoire de la communication que dans l'histoire de l'art.

Georges Didi-Huberman souligne que l'Iconologia de Ripa établit un lien étroit entre le visible et le lisible : l'image se donne à lire comme les mots d'un dictionnaire. En même temps, elle noue un trait semblable entre le visible et l'invisible, ainsi qu'en témoigne le prologue qui stipule : «Les images faites pour signifier une chose différente de celle que l'œil voit.... ${ }^{28}$. L'allégorie construit ainsi un schéma énonciatif sur le modèle «visible-lisible-invisible». Dans l'Iconologie de Gravelot, les figures entrent elles-mêmes dans des rapports binaires opposant symétriquement la Vertu au Vice (l'Abstinence - la Gourmandise) ou une figure positive et une négative (l'Allégresse - le Chagrin). Cette articulation syntagmatique qui sous-tend l'allégorie en fait moins une image qu'un langage ou plutôt ce que Christian Metz appelait un discours imagé.

Ce n'est cependant pas cette conception classique de l'allégorie qui est convoquée ici, même si l'on voit bien tout ce qui, dans Gribiche, fonctionne sur le mode allégorique : discours, lisibilité de l'image, fonction de désignation et de généralisation, didactisme et surtout le recours constant aux objets emblématiques (la serviette au col ou sur les genoux, tel le bandeau sur les yeux de la justice). Walter Benjamin, dans sa solide étude du Trauerspiel (le drame baroque allemand) et ensuite dans ses fragments sur Baudelaire ${ }^{29}$, fonde une véritable théorie moderne de l'allégorie. En associant l'histoire littéraire, la sociologie des productions culturelles et la philosophie de l'histoire, il quitte totalement l'approche interprétative de l'allégorie pour ne plus s'attacher qu'à sa fonction signifiante — soit au travail auquel elle procède - et à son inscription dans l'Histoire. Benjamin démontre que l'importance culturelle de l'allégorie dépasse le cadre étroit du signe auquel on l'a souvent assimilée. Selon lui, le symbole est «mystique» et l'allégorie «dialectique». Le premier est une transfiguration qui s'ouvre à l'expérience eschatologique, alors que la seconde relève de l'histoire de la nature et de l'homme. Le premier fait apparaitre l'infini dans ce qui est fini, la seconde rapporte le fini au fini . Ce passage est essentiel :

«Alors que dans le symbole, par la sublimation de la chute, le visage transfiguré de la nature se révèle fugitivement dans la lumière du salut, en revanche, dans l'allégorie, c'est la facies hippocratica de l'histoire qui s'offre au regard du spectateur comme un paysage primitif pétrifié. L'histoire, dans ce qu'elle a toujours eu d'intempestif, de douloureux, d'imparfait, s'inscrit dans un visage - non : dans une tête de mort. Et aussi vrai qu'il n'y a en celle-ci nulle liberté «symbolique» de l'expression, nulle harmonie classique de la forme, nulle humanité, l'énigme qui s'exprime dans cette figure, la plus soumise à l'empire de la nature, ce n'est pas simplement la nature de l'existence humaine, mais l'historicité de la biographie individuelle. C'est là le noyau de la vision allégorique, de l'exposition baroque de l'histoire comme histoire des souffrances du monde; elle n'a de signification que dans les stations de sa décadence.. ${ }^{30}$

Le symbole appartient à l'ordre du sublime (c'est-à-dire ce qui, dans l'ordre des choses et du monde, dépasse le sujet par sa puissance infinie et imperceptible) tandis que l'allégorie appartient à l'ordre visuel. Elle partage avec la vision «l'abîme qui sépare l'image et la signification». La distance conventionnelle qui spécifie l'allégorie est le lieu d'une expérience des limites du signe, des limites de l'histoire et des limites de l'homme. Elle est un langage qui exprime la finitude de l'histoire humaine.

L'allégorie, constate Benjamin, vieillit. Elle relève de conventions à la fois figées (par la règle qui les institue) et instables (en raison de leur vieillissement qui les fait tomber en désuétude).Son 
intérêt épistémologique réside dans l'inévitable historicité de sa forme, et donc dans ce qui, en elle, relève moins de l'émergence du sens que sa perte. L'allégorie, certes, renvoie à une idée, mais une idée toujours en manque de sens au regard de l'exégète à qui échoit la tâche, parfois difficile, de reconstitution des anciennes conventions. Toute «lecture» iconographique de l'allégorie procède toujours, un peu mélancoliquement, de la recherche du sens perdu. L'allégorie est donc moins une image qu'une ruine d'image dont l'interprétation, en grande partie archéologique, procède aussi de la reconstruction. C'est pourquoi, constate Benjamin, la figure allégorique suprême à l'âge baroque est la ruine:

«Avec elle, l'histoire s'est retirée sur le théâtre de manière sensible. Et dans cette forme, l'histoire n'est pas modelée, figurée comme le processus d'une vie éternelle, mais bien plutôt comme celui d'un déclin inéluctable. Ainsi l'allégorie reconnait-elle qu'elle est au-delà de la beauté. Les allégories sont au domaine de la pensée ce que les ruines sont au domaine des choses. D'où le culte baroque de la ruine.»1

L'allégorie est le théâtre de l'histoire, qui y expose, avec ostentation, son corps décharné, son cadavre, d'où toute vie s'est échappée. L'allégorie signifie la ruine, la perte, le détachement du sujet de l'expérience sensible, le déclin (thème benjaminien par excellence). Enfin, «la connaissance du caractère éphémère des choses, et le souci de les rendre éternelles, pour les sauven», est un de ses motifs les plus forts — dont la ruine est la figure-type. «[Elle] a sa demeure la plus durable à l'endroit où l'éphémère et l'éternel se touchent au plus près.»>

S'il ne manque pas d'images allégoriques classiques dans le cinéma des premiers temps (chez Zecca par exemple, à la fin de La grève : l'allégorie de la Justice signe la réconciliation du Travail et du Capital), elles perdent souvent leur fonction édificatrice traditionnelle, surtout chez Méliès qui joue déjà, ironiquement, de la facticité d'allégories tombées en désuétude. Avec le Mode de Représentation Institutionnel, l'allégorie sort des limites étroites de la figure unique (souvent le dernier plan dans les films des années 1910) pour se déployer dans l'espace et le temps de l'ensemble du récit. Dans cette histoire de l'allégorie filmique, le cinéma hollywoodien, principalement le film de genre (western, mélodrame ou fantastique), occupe une place majeure. De The Birth of a Nation de Griffith à The Day The Earth Stood Still de Wise, de The Half-Breed d'Allan Dwan à The Searchers de Ford, de Invasion of The Body Snatchers de Don Siegel à son remake récent par Ferrara, le cinéma américain a fait de l'allégorie le mode discursif le plus approprié à l'édification de l'identité nationale, raciale et culturelle des Etats-Unis autant qu'à la dénonciation de tout ce qui peut la menacer.

Cependant, l'intérêt d'une théorie moderne de l'allégorie n'est pas dans l'interprétation du discours caché de ce genre de film, à laquelle historiens et critiques procèdent plus ou moins bien depuis longtemps. Une telle théorie n'a d'intérêt qu'à proposer un concept esthétique apte à prendre en compte un ensemble de données qui ont caractérisés certains films au cours de l'histoire. Ces données, rappelons-le, sont les suivantes : l'assujettissement à diverses conventions (de genre par exemple), l'énonciation d'un discours (édificateur ou didactique) ou d'une idée supérieure supposée universelle, l'absence de recherche formelle et d'originalité (ce qui n'empêche pas la qualité du travail), la théâtralité (entendue au sens d'une exposition frontale et ostentatoire), enfin et surtout l'historicité des films, leur inévitable vieillissement et la perte de sens que cela entraîne. Depuis ses 
origines, le cinéma énonce des discours dont certains peuvent être devenus aujourd'hui presque hermétiques. C'est pour cette raison que certains esthéticiens n'en ont cure, estimant - avec raison - qu'un film ne se décrypte pas comme une vieille inscription. Ce que le concept d'allégorie vise dans un film, c'est la trace que l'histoire a laissée en lui, avec son cortège d'emblèmes énigmatiques; c'est la part de sens qui s'est perdue et l'a transformé en ruine, moins à cause du temps qui attaque la pellicule, qu'à cause de l'histoire qui fait disparaitre le sens dans le grand gouffre de l'oubli.

Ce qui, dans Gribiche, était immédiatement perceptible en 1925, ne l'est plus à présent et demande, pour être saisi, une longue exégèse. Les conventions culturelles permettaient au public de l'époque de comprendre ce que signifie un style, un objet ou une photo sur un mur. Bon nombre des signes ainsi dispersés dans le film ne sont peut-être même plus reconnaissables comme signes par un spectateur d'aujourd'hui. Même l'idée générale visée par le film (donner une image positive de la culture populaire) n'est plus perceptible, y compris aux yeux de certains historiens qui la manquent de peu ou de beaucoup. En général, le film est très peu commenté, signe qu'il ne "parle» plus guère et que son langage, trop daté sans doute, n'est déjà plus compréhensible. Sadoul l'écarte pour excès de sentimentalisme. Mitry n'y voit que le souci de «mettre en valeur le modernisme agressif de Süe et Mare» et déplore «l'allongement excessif d'une intrigue égarée dans le développement inutile de scènes sans objet» ${ }^{33}$. Ivo Blom écrit que Gribiche quitte volontairement «son taudis» et que «Feyder estime que la charité est une bonne chose tant qu'elle ne se fixe pas sur un enfant en particulier» ${ }^{34}$. Henri Fescourt, toujours pertinent et bien inspiré, a bien perçu les qualités du film, sans pour autant en saisir le véritable enjeu:

«Comment également ne pas apprécier le tableau d'un Paris de petits commerçants, d'ouvriers gagnant bien leur vie, de leurs mœurs franches, par exemple, le dîner à la brasserie, un dimanche soir, une de ces brasseries où il y a beaucoup de monde, où le garçon connaît les clients et où l'on mange bien. (...) Pour tant de raisons, Gribiche mériterait de sortir de son obscurité. Il eut la malchance d'être desservi par ses qualités. La somme de ses observations lassait. $\rangle^{35}$

Seul François Albéra, déjà cité, mesure bien la portée du film, même s’il ignore les signes visibles de la culture populaire dans l'appartement de Gribiche, qu'il qualifie de «banal». Quelque chose donc, dans ce film, semble s'être perdu, qu'il a fallu tant bien que mal reconstituer, comme au siècle dernier on reconstruisait les ruines pour épancher sa mélancolie. Cette perte du sens stigmatise de nombreux films voués à l'illisibilité ou, comme les hiéroglyphes à l'époque de la Renaissance, à orner le portail des érudits. C'est le prix que les allégories payent à l'histoire, et qu'il faut seulement inscrire au livre des comptes. Gribiche, comme tant d'autres allégories, s'offre aujourd'hui au regard du spectateur «comme un paysage primitif pétrifié».

Marc-Emmanuel Mélon

Centre de recherche sur les Arts du Spectacle, le cinéma et les arts visuels

Département des arts et sciences de la communication

Université de Liège.

Place du XX-Août, 7, 4000 Liège

Tél. 04/366 3255 ou 3279 (secrétariat)

ME.Melon@ulg.ac.be 
${ }^{1}$ Conférence de Jacques Feyder à la Tribune du Jeune Barreau de Bruxelles en juin 1938. Compte-rendu et retranscription partielle parus dans La Libre Belgique, 3 juin 1938.

${ }^{2}$ Charles FORD, Jacques Feyder, Paris, Seghers, coll. «Cinéma d'Aujourd'hui», 1973.

${ }^{3}$ Pierre BILLARD, L'âge classique du cinéma français. Du cinéma parlant à la Nouvelle Vague, Paris, Flammarion, 1995, p. 77.

${ }^{4}$ Henri FESCOURT, La Foi et les Montagnes ou le septième art au passé, Paris, Montel, 1959, p. 267.

${ }^{5}$ Nino FRANK, «Jacques Feyder», in Jacques Feyder ou le cinéma concret, Bruxelles, Comité National Jacques Feyder, Palais des Beaux-Arts, 1949, p. 16.

${ }^{6}$ Victor BACHY, Jacques Feyder, un artisan du cinéma, Louvain, Librairie Universitaire, 1968.

${ }^{7}$ La Libre Belgique, 3 juin 1938. Cette phrase est reprise, au mot près, dans Le cinéma, notre métier, Vésenaz, Genève, Pierre Caillé, 1946, p. 10.

${ }^{8}$ Jacques Feyder, «Souvenirs d'un cinéaste» in J. FEYDER et F.ROSAY, Le cinéma, notre métier, op cit, p. 7.

${ }^{9}$ Cité par Charles FORD, Jacques Feyder, op.cit., p.89.

${ }^{10}$ Ibidem.

${ }^{11}$ Pour un examen général de cette question de l'image et de la pensée, voir entre autres Jacques AUMONT, A quoi pensent les films ?, Paris, Séguier, 1996, en particulier le chapitre intitulé «Figurable, figuratif, figural», pp. 148173. La «figure» est sans doute le concept qui, dans les recherches actuelles, synthétise le mieux cette tendance théorique. Voir à ce propos les travaux de Nicole BRENEZ, dans un prochain ouvrage à paraître aux Editions De Boeck (Bruxelles) et de Philippe DUBOIS, notamment sa très belle étude de la figure de la Tempête chez Epstein («La Tempête et la matière-temps ou le Sublime et le Figural dans l'œuvre de Jean Epstein», à paraître dans les actes du colloque «Jean Epstein» (Paris, Cinémathèque française, automne 1998).

${ }^{12}$ Jean-Louis BAUDRY, «Effets idéologiques produits par l'appareil de base», repris in L'effet cinéma, Paris, Albatros, coll.«Ça-Cinéma», 1978, p. 20.

${ }^{13}$ Il semble que ce soit Françoise Rosay qui ait découvert le livre et l'ai proposé à Feyder. $C f$. l'autobiographie de Françoise Rosay, La traversée d'une vie ainsi que l'entretien qu'elle a accordé à Victor Bachy en 1966, in Les Cahiers de la Cinémathèque, $\mathrm{n}^{\circ} 40,1984, \mathrm{p}$. 9. Le film s'achève sur un plan de l'ouvrage publié aux éditions Gallimard, dans la collection «Cinario».

${ }^{14}$ Sur la compagnie Albatros, voir l'étude remarquable de François ALBERA, Albatros. Des Russes à Paris, 19191929, Paris, Milan, Cinémathèque française, Mazzotta, 1995.

${ }^{15}$ Ciné-Miroir, 1er septembre 1925. Cité par Georges SADOUL, Histoire Générale du Cinéma, vol. 5, Paris, Denoël, 1975, p. 186.

${ }^{16}$ Remarque déjà formulée par Lenny Borger à propos de Visages d'enfants, in Les Cahiers du muet, Bruxelles, Cinémathèque Royale de Belgique, 1993, nº 30.

${ }^{17}$ F. ALBERA, qui fait une courte mais pertinente analyse du film, suggère que cette scène parodie The Kid, de Chaplin, comme semble l'indiquer la présence d'une photo de Charlot accrochée au mur de la chambre de Gribiche (op.cit., p. 148).

${ }^{18}$ Comme l'a montré Zola dans Au Bonheur des dames . La structure duelle du film de Feyder n'est d'ailleurs pas sans analogie avec celle du roman.

${ }^{19}$ Charles BAUDELAIRE, «Le peintre de la vie moderne» (1863), repris in Ecrits sur l'art, Paris, Le Livre de Poche, 1992, p. 378.

${ }^{20}$ F. ALBERA, op.cit., pp. 145-148.

${ }^{21}$ Voir à ce sujet Giulia VERONESI, Style 1925, Paris, Lausanne, Anthony Krafft, 1968.

${ }^{22}$ Voir à ce sujet Molly NESBIT et Françoise REYNAUD, Eugène Atget. Intérieurs parisiens. Un album du Musée Carnavalet, Paris, Paris-Musées / Editions Carré, 1992; Molly NESBIT, Atget's Seven Albums, New Haven, Yale University Press, 1992.

${ }^{23}$ On se souvient du commentaire éclairant qu'en fit Walter Benjamin : «On a dit à juste titre qu'il avait phographié ces rues comme on photographie un théâtre du crime. Le théâtre du crime est, lui aussi, désert. Le cliché qu'on en prend n'a d'autre but que de déceler des indices. Pour l'évolution historique, ceux qu'a laissés Atget sont de 
véritables pièces à conviction. Aussi ont-ils une secrète signification politique. Ils exigent déjà qu'on les accueille en un certain sens. Ils ne se prêtent plus guère à une considération détachée. Ils inquiètent celui qui les regarde : pour les atteindre, le spectateur devine qu'il faut suivre une certaine voie.» Walter BENJAMIN, «L'œuvre d'art à l'ère de sa reproductibilité technique» (1935) in Poésie et révolution, Paris, Denoël, 1971, p. 185.

${ }^{24}$ Iconologie ou la Science des Emblèmes, devises, etc. Qui apprend à les expliquer, dessiner et inventer. Ouvrage très utile aux orateurs, poètes, peintres, sculpteurs, graveurs et généralement à toutes sortes de curieux des BeauxArts et des Sciences. Enrichie et augmentée d'un grand nombre de figures avec des moralités, tirées la plupart de Cesar Ripa. Par J.B. (= Jean Baudouin), de l’Académie Française, Amsterdam, 1698.

${ }^{25}$ Sur l'allégorie de la Justice, voir E. PANOFSKY, Essais d'iconologie (1939), Paris, Gallimard, 1967, p. 166 et surtout Robert JACOB, Images de la Justice. Essai sur l'iconographie judiciaire du Moyen Âge à l'âge classique, Paris, Le Léopard d'or, 1994, pp. 219-242.

${ }^{26}$ Cesare RIPA, Iconologia overo Descrittione dell'Imagini universali cavate dall'Antichità e da altri luoghi (...) per rappresentare le virtù, vitii, affetti, e passioni humane, Rome, 1593. Réed. New York, Londres, Garland, 1976.

${ }^{27}$ Par exemple celui de Jean Baudouin cité ci-dessus (Cf. note 21). En 1791 encore, H. GRAVELOT et COCHIN publient une Iconologie par figures ou Traité des Allégories qui se fonde sur le modèle du recueil de Ripa.

${ }^{28}$ Cesare RIPA, cité par Georges DIDI-HUBERMAN, Devant l'image, Paris, Minuit, 1990, p.146.

${ }^{29}$ Walter BENJAMIN, Origine du drame baroque allemand (1925), traduction Sybille Muller, Paris, Flammarion, 1985. «Zentralpark. Fragments sur Baudelaire», repris in Charles Baudelaire. Un poète lyrique à l'apogée du capitalisme, trad. Jean Lacoste, Paris, Payot, 1979.

${ }^{30}$ Ibid., p. 179.

${ }^{31}$ Ibid., p. 191.

${ }^{32}$ Ibid., pp. 241-242.

${ }^{33}$ Jean MITRY, Histoire du cinéma, tome III, Paris, Editions Universitaires, 1973, p. 374.

${ }^{34}$ Ivo BLOM, Les Cahiers du muet, Bruxelles, Cinémathèque Royale de Belgique, 1995, nº 16.

${ }^{35}$ Henri FESCOURT, La Foi et les Montagnes, op. cit., pp. 274-75. 